\title{
Variance-Constrained Control for Uncertain Stochastic Systems With Missing Measurements
}

\author{
Zidong Wang, Senior Member, IEEE, Daniel W. C. Ho, Senior Member, IEEE, and Xiaohui Liu
}

\begin{abstract}
In this paper, we are concerned with a new control problem for uncertain discrete-time stochastic systems with missing measurements. The parameter uncertainties are allowed to be norm-bounded and enter into the state matrix. The system measurements may be unavailable (i.e., missing data) at any sample time, and the probability of the occurrence of missing data is assumed to be known. The purpose of this problem is to design an output feedback controller such that, for all admissible parameter uncertainties and all possible incomplete observations, the system state of the closed-loop system is mean square bounded, and the steady-state variance of each state is not more than the individual prescribed upper bound. We show that the addressed problem can be solved by means of algebraic matrix inequalities. The explicit expression of the desired robust controllers is derived in terms of some free parameters, which may be exploited to achieve further performance requirements. An illustrative numerical example is provided to demonstrate the usefulness and flexibility of the proposed design approach.
\end{abstract}

Index Terms-Algebraic matrix inequality, incomplete observation, missing signal, robust control, stochastic control, variance constraints.

\section{INTRODUCTION}

$\mathbf{I}$ $\mathrm{T}$ IS often the case in many practical stochastic control problems that, the performance requirements are naturally expressed in terms of the upper bounds on the steady-state variances [19]. For example, in an aiming control problem, the residence time in a given pointing direction (within circle of radius $R$ ) is inversely proportional to the maximum eigenvalue of the covariance matrix [10], and therefore the variance upper bounds are directly related to the aiming control performances. Traditional control design techniques, such as LQG and $H_{\infty}$ control theories, cannot be directly applied in this kind of design problems. For instance, the LQG controllers minimize a linear quadratic performance index, which lacks guaranteed variance constraints with respect to individual system states.

Manuscript received February 12, 2004; revised May 24, 2004. This work was supported in part by the Engineering and Physical Sciences Research Council (EPSRC), U.K. under Grant GR/S27658/01, in part by the Nuffield Foundation, U.K. under Grant NAL/00630/G, in part by the Research Grants Council (RGC) of Hong Kong under Grant CityU/101103, and in part by the Alexander von Humboldt Foundation of Germany. This paper was recommended by Associate Editor G. C. Calafiore.

Z. Wang is with the School of Information Sciences and Technology, Donghua University, Shanghai 200051, China, and also with the Department of Information Systems and Computing, Brunel University, Middlesex UB8 3PH, U.K. (e-mail: Zidong.Wang@ @runel.ac.uk).

$\mathrm{X}$. Liu is with the Department of Information Systems and Computing, Brunel University, Middlesex UB8 3PH, U.K.

D. W. C. Ho is with Department of Mathematics, City University of Hong Kong, Kowloon, Hong Kong.

Digital Object Identifier 10.1109/TSMCA.2005.851124
On the other hand, the covariance control theory has provided a more direct methodology for achieving the individual variance constraints than the LQG control theory, see [19] and references therein. The main idea of the covariance control theory is to choose a state covariance according to different requirements on the system performance and robustness, and then to design a controller so that the specified state covariance is assigned to the closed-loop system. The covariance control design follows these three steps [19]; 1) formulate the control design objectives as constraints in the space of covariance matrices, 2) use numerical techniques to obtain an assignable covariance that satisfies the design objectives and, 3) parameterize the set of all controllers that assign the desired covariance, and obtain a satisfactory controller from this set, according to some given criteria.

Covariance control theory has been successfully applied in dealing with the pointing control problem for NASA's ACES structure such as the Hubble Space Telescope [29]. In the presence of model parameter uncertainties, the robust variance-constrained control problem has received considerable research attention, where the upper bounds are stated as design objectives imposed on the variance values, see e.g., [4], [8], [22], [26]. Also, the dual robust filtering problems with error variance constraints have recently gained initial research interest [24], [25], [27].

It should be pointed out that, in all the literature mentioned above, it is implicitly assumed that the measurements always contain the signal, which can be used for the feedback control. However, in practical applications such as target tracking control, there may be a nonzero probability that any observation consists of noise alone if the target is absent, i.e., the measurements are not consecutive but contain missing observations. In such a case, the missing data phenomena must be taken into account when designing the control law. Unfortunately, if some of the measurements are unavailable, the standard definition of covariance in the data statistical analysis does not directly apply. The conventional optimal (robust, $H_{\infty}$ ) control theories, which are dependent on the system output covariance, do not suit the case when there are missing measurements. Hence, it is important to develop a new stochastic control approach to tackling the missing measurement problem.

The missing measurements are caused by a variety of reasons, e.g., the high maneuverability of the tracked target, a certain failure in the measurement, intermittent sensor failures, accidental loss of some collected data, or some of the data may be jammed or coming from a high noise environment, etc., see [13]. Missing data analysis problem is well understood and has been studied extensively in the context of time series analysis and system identification (see [3], [17] and the references therein), 
where the research attention has been focused on the statistical inference and modeling of missing data. In particular, there exists a great deal of literature on the study of the statistical law of the missing data. Recently, the robust filtering problem with missing data has been investigated in [15], [16], and [23].

Naturally, after the system model with missing measurement is built up, the corresponding control problem should then be considered, probably with relaxed performance requirements. For example, when tracking a highly maneuverable target, it is important to control the tracking system such that the tracking error is bounded in the mean square in the presence of missing measurements. Similar problems also exist in other engineering applications, such as the network congestion control when there are signals missing during transmission, where the missing observation is also named as dropout or intermittence, see [7], [9], and [18]. So far, to the best of the authors' knowledge, the control problem for stochastic systems with or without uncertainties under missing measurements has not been fully investigated and remains to be important and challenging.

In this paper, we are concerned with a new control problem for uncertain discrete-time stochastic systems with missing measurements The parameter uncertainties are allowed to be norm-bounded and enter into the state matrix. The system measurements may be unavailable (i.e., missing data) at any sample time, and the probability of the occurrence of missing data is assumed to be known. The purpose of this problem is to design an output feedback controller such that, for all admissible parameter uncertainties and all possible incomplete observations, the system state of the closed-loop system is mean square bounded, and the steady-state variance of each state is not more than the individual prescribed upper bound. We show that the addressed problem can be solved in terms of algebraic matrix inequalities, and derive the explicit expression of the desired robust controllers. An illustrative numerical example is provided to demonstrate the usefulness and flexibility of the proposed design approach.

Notation: The notations in this paper are quite standard. $\mathbb{R}^{n}$ and $\mathbb{R}^{n \times m}$ denote, respectively, the $n$ dimensional Euclidean space and the set of all $n \times m$ real matrices. The superscript " $T$ " denotes the transpose and the notation $X \geq Y$ (respectively, $X>Y$ ) where $X$ and $Y$ are symmetric matrices, means that $X-Y$ is positive semi-definite (respectively, positive definite). $I$ is the identity matrix with compatible dimension. Let $\left(\Omega, \mathcal{F},\left\{\mathcal{F}_{t}\right\}_{t \geq 0}, P\right)$ be a complete probability space with a filtration $\left\{\mathcal{F}_{t}\right\}_{t>0}$ satisfying the usual conditions (i.e., the filtration contains all $P$-null sets and is right continuous). $\mathcal{E}\{\cdot\}$ stands for the mathematical expectation operator with respect to the given probability measure $P$. Prob $\{\cdot\}$ means the occurrence probability of the event ".". For a real matrix $B, B^{+}$denotes its Moore-Penrose inverse. Sometimes, the arguments of a function will be omitted in the analysis when no confusion can arise.

\section{Problem Formulation AND Assumptions}

Consider the following uncertain discrete-time stochastic system:

$$
x(k+1)=(A+\Delta A) x(k)+B u(k)+w(k)
$$

and the measurement equation

$$
y(k)=\gamma(k) C x(k)+v(k)
$$

where $x \in \mathbb{R}^{n}$ is a state vector, $u \in \mathbb{R}^{m}$ is a control input vector, $y \in \mathbb{R}^{p}$ is a measured output vector, and $A, B$ and $C$ are known constant matrices. $w(k) \in \mathbb{R}^{n}$ and $v(k) \in \mathbb{R}^{p}$ are mutually uncorrelated zero mean Gaussian white noise sequences with respective covariances $W>0$ and $V>0$. The initial state $x(0)$ has the mean $\bar{x}(0)$ and covariance $X(0)$, and is uncorrelated with both $w(k)$ and $v(k) . \Delta A$ is a real-valued perturbation matrix that represents parametric uncertainty being of the following form:

$$
\Delta A=M F N, \quad F F^{T} \leq I
$$

and $M$ and $N$ are known constant matrices of appropriate dimensions which specify how the elements of the nominal matrix $A$ are affected by the uncertain parameters in $F$. The uncertainties in $\Delta A$ are said to be admissible if (3) holds.

The stochastic variable $\gamma(k) \in \mathbb{R}$ is assumed to be a Bernoulli distributed white sequence taking values on 0 and 1 with

$$
\operatorname{Prob}\{\gamma(k)=1\}=\mathcal{E}\{\gamma(k)\}:=\bar{\gamma}
$$

where $\bar{\gamma}$ is a known positive constant, and $\gamma(k) \in \mathbb{R}$ is assumed to be independent of $w(k), v(k)$, and $x(0)$. Therefore, we have

$$
\begin{aligned}
\operatorname{Prob}\{\gamma(k)=0\} & =1-\bar{\gamma} \\
\sigma_{\gamma}^{2}:=\mathcal{E}\left\{(\gamma(k)-\bar{\gamma})^{2}\right\} & =(1-\bar{\gamma}) \bar{\gamma} .
\end{aligned}
$$

Remark 1: The system measurement mode (2) was first introduced in [11], and has then been used in many papers (see e.g., [12], [23]) to account for the probabilistic measurement missing. The corresponding probability $\bar{\gamma}$ could be estimated through statistical tests.

Remark 2: The parameter uncertainty described in (3) has been frequently employed in dealing with robust filtering and control problems for uncertain systems, see e.g., [24], since many practical systems possess parameter uncertainties which can be either exactly modeled or overbounded by (3). It is noted that the unknown matrix $F$ in (3) can even be allowed to be time-varying and state-dependent, i.e., $F=F(t, x(t))$, as long as (3) is satisfied.

For technical convenience, we define a new stochastic sequence

$$
\tilde{\gamma}(k):=\gamma(k)-\bar{\gamma}
$$

It is not difficult to see that $\tilde{\gamma}(k)$ is a scalar zero mean stochastic sequence with variance

$$
\sigma_{\tilde{\gamma}}^{2}=(1-\bar{\gamma}) \bar{\gamma}
$$

When an output feedback control law

$$
u(k)=K y(k)
$$

is applied to the system (1) and (2), the closed-loop system is governed by

$$
\begin{array}{r}
x(k+1)=(A+\bar{\gamma} B K C+\Delta A) x(t)+\tilde{\gamma}(k) B K C x(k) \\
+B K v(k)+w(k) .
\end{array}
$$


Remark 3: It should be pointed out that a stochastic variable $\tilde{\gamma}(k)$ is contained in (10), which reflects the characteristic of the missing measurement for the addressed control problem. Hence, the system (10) is actually a stochastic parameter system, for which the performance analysis issue would become involved.

The steady-state state covariance is defined by

$$
X:=\lim _{k \rightarrow \infty} X(k):=\lim _{k \rightarrow \infty} \mathcal{E}\left[x(k) x^{T}(k)\right] .
$$

Using the statistics of the stochastic sequences $w(k), v(k)$ and $\tilde{\gamma}(k)$, we can find that the state covariance $X(k)$ defined in (11) satisfies

$$
\begin{aligned}
X(k+1) & =(A+\bar{\gamma} B K C+\Delta A) X(k)(A+\bar{\gamma} B K C+\Delta A)^{T} \\
& +\sigma_{\tilde{\gamma}}^{2}(B K C) X(k)(B K C)^{T} \\
& +B K V K^{T} B^{T}+W .
\end{aligned}
$$

We know from [1] and [5] that, if the state of the system (10) is mean square bounded, then the steady-state covariance $X$ defined in (11) exists and satisfies the following discrete-time modified Lyapunov equation:

$$
\begin{aligned}
X= & (A+\bar{\gamma} B K C+\Delta A) X(A+\bar{\gamma} B K C+\Delta A)^{T} \\
& +\sigma_{\tilde{\gamma}}^{2}(B K C) X(k)(B K C)^{T} \\
& +B K V K^{T} B^{T}+W .
\end{aligned}
$$

Remark 4: It follows from [1] and [5] that, there exists a unique symmetric positive semi-definite solution to (13) if and only if

$$
\begin{aligned}
\rho\{(A+\bar{\gamma} B K C+\Delta A) \otimes & (A+\bar{\gamma} B K C+\Delta A) \\
& \left.+\sigma_{\tilde{\gamma}}^{2}(B K C) \otimes(B K C)\right\}<1
\end{aligned}
$$

where $\rho$ is the spectral radius and $\otimes$ is the Kronecker product. Furthermore, we also know from [1], [5] that the condition (14) is equivalent to the mean square boundedness of the state of the system (10). To this end, we achieve the conclusion that, if there exists a positive definite solution to the (13), then (14) holds, and the state covariance $X(k)$ defined in (11) will converge to its steady-state value $X$.

The purpose of this paper is to design the controller gain $K$ such that in the presence of missing measurement as well as all admissible perturbations $\Delta A$, the following two performance requirements are simultaneously satisfied:

1) The state of the system (10) is mean-square bounded, i.e., (14) holds.

2) The steady-state covariance $X$ exists and satisfies

$$
[X]_{i i} \leq \alpha_{i}^{2}, \quad i=1,2, \ldots, n .
$$

where $[X]_{i i}$ means the steady-state variance of the $i$ th state, and $\alpha_{i}^{2}(i=1,2, \ldots, n)$ denotes the prespecified steady-state variance constraint on the $i$ th state.

In Section III, we will give a solution to the problem addressed above. Specifically, we will first characterize an upper bound on the steady-state covariance $X$ satisfying (13), let this upper bound meet the prespecified variance constraints, and then we will parameterize all desired controller gains with which the resulting steady-state state covariance is not more than the obtained upper bound.

\section{MAIN Results AND PROOFS}

We will need the following lemmas to establish our main results.

Lemma 1: [21] Let a positive scalar $\varepsilon>0$ and a positive definite matrix $Q>0$ be such that

$$
N Q N^{T}<\varepsilon I \text {. }
$$

Assume that $\triangle A=M F N$ where $F F^{T} \leq I$, and $M$ and $N$ are constant matrices with appropriate dimensions. Then

$$
\begin{aligned}
\left(A_{n}+\Delta A\right) & Q\left(A_{n}+\Delta A\right)^{T} \\
& \leq A_{n}\left(Q^{-1}-\varepsilon^{-1} N^{T} N\right)^{-1} A_{n}^{T}+\varepsilon M M^{T}
\end{aligned}
$$

holds for all admissible perturbations $\Delta A$.

Lemma 2: [6] Let $Y \in \mathbb{R}^{m \times p}(m \leq p)$ and $Z \in \mathbb{R}^{m \times n}$. There exists a matrix $U$ that satisfies simultaneously

$$
Y=Z U, \quad U U^{T}=I
$$

if and only if

$$
Y Y^{T}=Z Z^{T}
$$

In this case, a general solution for $U$ can be expressed as

$$
U=U_{Z}\left[\begin{array}{cc}
I & 0 \\
0 & T
\end{array}\right] U_{Y}^{T}, \quad U \in \mathbb{R}^{\left(n-r_{Z}\right) \times\left(p-r_{Z}\right)}, \quad T T^{T}=I
$$

where $U_{Z}$ and $U_{Y}$ come from the singular value decomposition of $Z$ and $Y$, respectively

$$
\begin{aligned}
Z & =T_{Z}\left[\begin{array}{cc}
H_{Z} & 0 \\
0 & 0
\end{array}\right] U_{Z}^{T} \\
& =\left[\begin{array}{ll}
T_{Z 1} & T_{Z 2}
\end{array}\right]\left[\begin{array}{cc}
H_{Z} & 0 \\
0 & 0
\end{array}\right]\left[\begin{array}{l}
U_{Z 1}^{T} \\
U_{Z 2}^{T}
\end{array}\right] \\
Y & =T_{Y}\left[\begin{array}{cc}
H_{Y} & 0 \\
0 & 0
\end{array}\right] U_{Y}^{T} \\
& =\left[\begin{array}{ll}
T_{Y 1} & T_{Y 2}
\end{array}\right]\left[\begin{array}{cc}
H_{Y} & 0 \\
0 & 0
\end{array}\right]\left[\begin{array}{c}
U_{Y 1}^{T} \\
U_{Y 2}^{T}
\end{array}\right]
\end{aligned}
$$

and

$$
r_{Z}=\operatorname{rank}(Z), \quad T_{Z}=T_{Y}, \quad H_{Z}=H_{Y}
$$

For presentation convenience, we denote

$$
\begin{aligned}
\Phi:= & \left(Q^{-1}-\varepsilon^{-1} N^{T} N\right)^{-1} \\
R:= & \bar{\gamma}^{2} C \Phi C^{T}+\sigma_{\tilde{\gamma}}^{2} C Q C^{T}+V \\
\Omega:= & Q-A \Phi A^{T}-\varepsilon M M^{T}-W \\
& +\bar{\gamma}^{2} A \Phi C^{T} R^{-1} C \Phi A^{T} \\
Y:= & \bar{\gamma}\left(I-B B^{+}\right) A \Phi C^{T} R^{-1 / 2} \\
Z:= & \left(I-B B^{+}\right) \Omega^{1 / 2}
\end{aligned}
$$

where $\bar{\gamma}$ and $\sigma_{\tilde{\gamma}}$ are defined in (4) and (8), respectively.

The following theorem provides the conditions for the existence of a solution to the addressed variance-constrained control problem with missing measurements, which are derived by 
using the singular value decomposition technique and the generalized inverse theory.

Theorem 1: If there exist a positive scalar $\varepsilon>0$ and a positive definite matrix $Q>0$ such that (25)-(27), shown at the bottom of the page hold, where $\Phi$ is defined in (20) and $B^{+}$denotes the Moore-Penrose inverse of $B$, then there exists a feedback gain, $K$, such that the state of the system (10) is mean square bounded, and the steady-state covariance $X$ exists and satisfies $X \leq Q$.

Proof: It follows immediately from the matrix inverse lemma that

$$
\left(Q^{-1}-\varepsilon^{-1} N^{T} N\right)^{-1}=Q+Q N^{T}\left(\varepsilon I-N Q N^{T}\right)^{-1} N Q
$$

and therefore the inequality in (25), shown at the bottom of the page, implies that $\Phi>0$. Also, from the definition of $\Omega$ in (22), we know that the inequality in (26), shown at the bottom of the page, indicates $\Omega \geq 0$, and that (27), shown at the bottom of the page, can be rewritten as

$$
\left(I-B B^{+}\right)\left(\Omega-\bar{\gamma}^{2} A \Phi C^{T} R^{-1} C \Phi A^{T}\right)\left(I-B B^{+}\right)=0
$$

or

$$
Y Y^{T}=Z Z^{T}
$$

where $Y$ and $Z$ are defined in (23) and (24), respectively.

It follows from Lemma 2 that, there exists an orthogonal matrix $U$ (i.e., $U U^{T}=I$ ) such that

$$
Y=Z U
$$

which can be easily rearranged as

$$
\left(I-B B^{+}\right)\left(\Omega^{1 / 2} U R^{-1 / 2}-\bar{\gamma} A \Phi C^{T} R^{-1}\right)=0 .
$$

It is noticed from [2] that, the relation (31) is the necessary and sufficient condition for the existence of a solution $K$ satisfying

$$
B K=\Omega^{1 / 2} U R^{-1 / 2}-\bar{\gamma} A \Phi C^{T} R^{-1}
$$

which is equivalent to

$$
B K R^{1 / 2}+\bar{\gamma} A \Phi C^{T} R^{-1 / 2}=\Omega^{1 / 2} U .
$$

Since $U$ is an orthogonal matrix, it follows again from Lemma 2 that, (33) leads to

$$
\begin{aligned}
\Omega= & \left(\Omega^{1 / 2} U\right)\left(\Omega^{1 / 2} U\right)^{T} \\
= & \left(B K R^{1 / 2}+\bar{\gamma} A \Phi C^{T} R^{-1 / 2}\right) \\
& \cdot\left(B K R^{1 / 2}+\bar{\gamma} A \Phi C^{T} R^{-1 / 2}\right)^{T} \\
= & (B K) R(B K)^{T}+\bar{\gamma} B K C \Phi A^{T} \\
& +\bar{\gamma} A \Phi C^{T}(B K)^{T}+\bar{\gamma}^{2} A \Phi C^{T} R^{-1} C \Phi A^{T} .
\end{aligned}
$$

Substituting the definition (22) of $\Omega$ into (34), we obtain that

$$
\begin{aligned}
Q=A \Phi A^{T}+\varepsilon M M^{T}+W+\bar{\gamma} A \Phi C^{T}(B K)^{T} \\
+\bar{\gamma}(B K) C \Phi A^{T}+(B K) R(B K)^{T}
\end{aligned}
$$

Moreover, substituting (20) and (21) into (35) and performing straightforward manipulations result in

$$
\begin{aligned}
Q= & (A+\bar{\gamma} B K C)\left(Q^{-1}-\varepsilon^{-1} N^{T} N\right)^{-1}(A+\bar{\gamma} B K C)^{T} \\
& +\varepsilon M M^{T}+\sigma_{\tilde{\gamma}}^{2}(B K C) Q(B K C)^{T} \\
& +B K V K^{T} B^{T}+W .
\end{aligned}
$$

Next, we define

$$
\begin{aligned}
\Xi:= & (A+\bar{\gamma} B K C)\left(Q^{-1}-\varepsilon^{-1} N^{T} N\right)^{-1}(A+\bar{\gamma} B K C)^{T} \\
& +\varepsilon M M^{T}-(A+\bar{\gamma} B K C+\Delta A) Q \\
& \cdot(A+\bar{\gamma} B K C+\Delta A)^{T}
\end{aligned}
$$

and know from Lemma 1 that $\Xi \geq 0$. To this end, it follows from (36) and (37) that, there exist a positive definite matrix $Q>0$ and a feedback gain $K$ such that

$$
\begin{aligned}
Q= & (A+\bar{\gamma} B K C+\Delta A) Q(A+\bar{\gamma} B K C+\Delta A)^{T} \\
& +\sigma_{\tilde{\gamma}}^{2}(B K C) Q(B K C)^{T}+B K V K^{T} B^{T} \\
& +W+\Xi .
\end{aligned}
$$

Subsequently, we have

$$
\begin{aligned}
&(A+\bar{\gamma} B K C+\Delta A) Q(A+\bar{\gamma} B K C+\Delta A)^{T} \\
&-Q+\sigma_{\tilde{\gamma}}^{2}(B K C) Q(B K C)^{T}<0
\end{aligned}
$$

which leads to (14). As discussed in Remark 4, we know that the state of the system (10) is mean square bounded, and the steady-state covariance $X$ exists and satisfies (13).

Finally, subtract (13) from (38) to give

$$
\begin{aligned}
& (A+\bar{\gamma} B K C+\Delta A)(Q-X)(A+\bar{\gamma} B K C+\Delta A)^{T} \\
& -(Q-X)+\sigma_{\tilde{\gamma}}^{2}(B K C)(Q-X)(B K C)^{T}=-\Xi \leq 0
\end{aligned}
$$

which indicates again from Remark 4 that $Q-X \geq 0$. This completes the proof of this theorem.

Remark 5: It is evident from Theorem 1 that, if there exist $\varepsilon>0$ and $Q>0$ satisfying (25)-(27) as well as

$$
[Q]_{i i} \leq \alpha_{i}^{2}, \quad i=1,2, \ldots, n
$$

then there exists an output feedback gain $K$ such that: 1) the state of the closed-loop system (10) is mean square bounded, and 2) the steady-state covariance $X$ exists and satisfies

$$
[X]_{i i} \leq[Q]_{i i} \leq \alpha_{i}^{2}, \quad i=1,2, \ldots, n .
$$

Hence, the design problem of variance-constrained output feedback control with missing measurements will be feasible.

$$
\begin{aligned}
N Q N^{T} & <\varepsilon I \\
Q-A \Phi A^{T}-\varepsilon M M^{T}-W+\bar{\gamma}^{2} A \Phi C^{T} R^{-1} C \Phi A^{T} & \geq 0 \\
\left(I-B B^{+}\right)\left(Q-A \Phi A^{T}-\varepsilon M M^{T}-W\right)\left(I-B B^{+}\right) & =0
\end{aligned}
$$


Remark 6: The results of Theorem 1 may be conservative due to the use of the inequalities (16). However, such conservatism can be significantly reduced by appropriate choices of the parameter $\varepsilon>0$ in a matrix norm sense. The relevant discussion and corresponding numerical algorithm can be found in [28] and references therein.

Having established the existence conditions of the desired feedback gains, our next goal is obviously to characterize the set of these gains. First, we introduce the singular value decompositions (18) and (19), where $Y, Z$ are defined in (23) and (24), respectively.

Suppose now that the conditions of Theorem 1 are satisfied. It follows from [2] that a general solution to (32) is given by

$$
K=B^{+}\left(\Omega^{1 / 2} U R^{-1 / 2}-\bar{\gamma} A \Phi C^{T}\right)+\left(I-B^{+} B\right) \Psi
$$

where $\Psi \in \mathbb{R}^{m \times p}$ is arbitrary, $U$ is any orthogonal matrix satisfying $Y=Z U$ and can be expressed, by Lemma 2, as

$$
U=U_{Z}\left[\begin{array}{cc}
I & 0 \\
0 & T
\end{array}\right] U_{Y}^{T}
$$

where

$$
U \in \mathbb{R}^{\left(n-r_{Z}\right) \times\left(p-r_{Z}\right)}, \quad T T^{T}=I
$$

and the orthogonal matrix $T$ is arbitrary, $r_{Z}=\operatorname{rank}(Z)$.

Summing up the above results, we are now in a position to characterize a set of desired controller gains satisfying (32). The following theorem can be easily proven by substituting (43) into (42).

Theorem 2: Assume that the conditions of Theorem 1 are satisfied. Then the set of all controller gains satisfying (32) is parameterized by

$$
\begin{aligned}
& \mathcal{K}=\left\{K: K=B^{+}\left(\Omega^{1 / 2} U_{Z}\left[\begin{array}{ll}
I & 0 \\
0 & T
\end{array}\right] U_{Y}^{T} R^{-1 / 2}\right.\right. \\
&\left.\left.-\bar{\gamma} A \Phi C^{T}\right)+\left(I-B^{+} B\right) \Psi\right\}
\end{aligned}
$$

where $\varepsilon>0$ and $Q>0$ satisfy (25)-(27), $\Omega$ is defined in (22), $U_{Y}$ and $U_{Z}$ come from the singular value decomposition of $Y$ and $Z$ in (18) and (19), $\Psi \in \mathbb{R}^{m \times p}$ is arbitrary, and $T \in$ $\mathbb{R}^{\left(n-r_{Z}\right) \times\left(p-r_{Z}\right)}$ is arbitrary orthogonal, $r_{Z}=\operatorname{rank}(Z)$.

Remark 7: It should be mentioned that, in the design of the controller gains, if the solution set is not empty, there exists much explicit freedom, such as the choices of the free parameter $\Psi \in \mathbb{R}^{m \times p}$ and the orthogonal matrix $T \in \mathbb{R}^{\left(n-r_{Z}\right) \times\left(p-r_{Z}\right)}$. This freedom does not affect the mean square boundedness of the closed-loop system, but could be potentially utilized to improve other system performances such as reliability behavior. The main results of this paper can also be extended to the case when there are some deterministic parameter uncertainties on the system outputs. The reason why we discuss the case when the parameter uncertainties only enter into the state matrix is just to make our theory more understandable and to avoid unnecessarily complicated notations.
The following corollary, which can be easily obtained from Theorem 1, Theorem 2, and Remark 5, solves the variance-constrained robust output feedback control problem with missing measurements addressed in Section II.

Corollary 1: If there exist a positive scalar $\varepsilon>0$ and a positive definite matrix $Q>0$ such that (25)-(27) hold, and $Q>0$ satisfies $[Q]_{i i} \leq \alpha_{i}^{2}(i=1,2, \ldots, n)$, then the controller (9) with the gain matrix $K$ determined by (44) will achieve the desired robust control performance for uncertain systems with missing measurements.

Remark 8: In practical applications, the designers would wish to construct $\varepsilon>0$ and matrix $Q>0$, which solve (26), subject to the constraints (25), (27) and (41), and then obtain the desired feedback gains from (44) readily. The condition (26) is actually quadratic matrix inequality (QMI), which could be dealt with by using the algorithms proposed in [14]. Also, for relatively lower-order models, the parameterization approach can be used to deal with the addressed matrix inequalities/equation, which will be demonstrated in Section IV. One of the future research topics would be to utilize the linear matrix inequality (LMI) approach for accommodating various desired performance requirements within the same LMI framework, since LMIs can be readily solved using LMI toolbox in Matlab.

\section{AN EXTENSION}

The aim of this section is to show that it is straightforward to extend the main results of the previous section to the dynamic output feedback case, which would facilitate the engineering applications.

Consider the following uncertain discrete-time stochastic system

$$
x_{p}(k+1)=\left(A_{p}+\Delta A_{p}\right) x_{p}(k)+B_{p} u(k)+w_{p}(k)
$$

and the measurement equation

$$
z(k)=\gamma(k) C_{p} x_{p}(k)+v_{p}(k)
$$

where $x \in \mathbb{R}^{n_{p}}$ is a state vector, $u \in \mathbb{R}^{m_{p}}$ is a control input vector, $z \in \mathbb{R}^{p_{p}}$ is a measured output vector, and $A_{p}, B_{p}$ and $C_{p}$ are known constant matrices. $w_{p}(k) \in \mathbb{R}^{n_{p}}$ and $v_{p}(k) \in \mathbb{R}^{p_{p}}$ are mutually uncorrelated zero-mean Gaussian white noise sequences with respective covariances $W_{p}>0$ and $V_{p}>0$. The initial state $x_{p}(0)$ has the mean $\bar{x}_{p}(0)$ and covariance $X_{p}(0)$, and is uncorrelated with both $w_{p}(k)$ and $v_{p}(k) . \Delta A_{p}$ is an uncertain matrix satisfying

$$
\Delta A_{p}=M_{p} F_{p} N_{p}, \quad F_{p} F_{p}^{T} \leq I
$$

and $M_{p}$ and $N_{p}$ are known constant matrices of appropriate dimensions. Again, the stochastic variable $\gamma(k) \in \mathbb{R}$ is a Bernoulli distributed white sequence taking values on 0 and 1 with

$$
\operatorname{Prob}\{\gamma(k)=1\}=\mathcal{E}\{\gamma(k)\}:=\bar{\gamma}
$$

where $\bar{\gamma}$ is a known positive constant, and $\gamma(k) \in \mathbb{R}$ is independent of $w_{p}(k), v_{p}(k)$, and $x_{p}(0)$. 
We consider a dynamic controller of order $n_{c}$ with state-space representation

$$
\begin{aligned}
x_{c}(k+1) & =A_{c} x_{c}(k)+B_{c} z(k), \\
u(k) & =C_{c} x_{c}(k)+D_{c} z(k) .
\end{aligned}
$$

It is expected to design a dynamic controller (49) and (50) such that, for all parameter uncertainties and all possible incomplete observations, the system state of the closed-loop system is mean square bounded, and the steady-state variance of each state is not more than the individual prescribed upper bound.

Combining (45), (46), (49), and (50), the closed-loop system can be expressed as the following augmented system:

$$
x(k+1)=(A+\gamma(k) B K C+\Delta A) x(t)+B K v(k)+w(k)
$$

where

$$
\begin{aligned}
x(k) & :=\left[\begin{array}{l}
x_{p}(k) \\
x_{c}(k)
\end{array}\right], \quad y(k):=\left[\begin{array}{c}
z(k) \\
x_{c}(k)
\end{array}\right] \\
A & :=\left[\begin{array}{cc}
A_{p} & 0 \\
0 & 0
\end{array}\right], \quad B:=\left[\begin{array}{cc}
B_{p} & 0 \\
0 & I
\end{array}\right] \\
C & :=\left[\begin{array}{cc}
C_{p} & 0 \\
0 & I
\end{array}\right], \quad K:=\left[\begin{array}{ll}
D_{c} & C_{c} \\
B_{c} & A_{c}
\end{array}\right] \\
M & :=\left[\begin{array}{c}
M_{p} \\
0
\end{array}\right], \quad F:=F_{p} \\
N & :=\left[\begin{array}{cc}
N_{p} & 0
\end{array}\right], \quad \Delta A:=M F N, \\
w(k) & :=\left[\begin{array}{c}
w_{p}(k) \\
0
\end{array}\right] \quad v(k):=\left[\begin{array}{c}
v_{p}(k) \\
0
\end{array}\right] .
\end{aligned}
$$

Note that (51) has the same form as (10). Therefore, our main results can be readily extended to the dynamical output feedback controller design case for uncertain stochastic systems with missing measurements and variance constraints.

\section{A NumericAl EXAMPLE}

In this section, we demonstrate the theory developed in this note by means of a simple example.

We consider an uncertain discrete-time stochastic system (1) and (2) with parameters given by

$$
\begin{aligned}
A & =\left[\begin{array}{cc}
1.5 & 0.1 \\
0.1 & -0.5
\end{array}\right], \quad B=\left[\begin{array}{l}
1 \\
0
\end{array}\right] \\
C & =\left[\begin{array}{ll}
1 & 0 \\
0 & 1
\end{array}\right], \quad M=\left[\begin{array}{cc}
0.1 & 0.05 \\
-0.02 & 0.8
\end{array}\right] \\
N & =\left[\begin{array}{cc}
0.1 & 0 \\
0 & 0.1
\end{array}\right], \quad W=\left[\begin{array}{cc}
0.1 & 0 \\
0 & 0.1
\end{array}\right] \\
V & =\left[\begin{array}{cc}
0.5 & 0 \\
0 & 0.5
\end{array}\right]
\end{aligned}
$$

and the probability for complete observation is assumed to be 0.9 , i.e., the missing probability is 0.1 .

The purpose of this example is to design the output feedback gain, $K$, such that for the missing measurements with given missing law as well as all admissible perturbations $\Delta A$, the state of the system (10) is mean square bounded, and the steady-state covariance $X$ exists and satisfies

$$
[X]_{11} \leq 2, \quad[X]_{22} \leq 1
$$

Now, it is supposed that the positive definite matrix $Q$ has the form $Q=\operatorname{diag}\left\{q_{11}, q_{22}\right\}$. Note that (27) reduces to the following:

$$
\begin{array}{r}
q_{22}-\left[0.01\left(q_{11}^{-1}-0.01 \varepsilon^{-1}\right)^{-1}+0.25\left(q_{22}^{-1}-0.01 \varepsilon^{-1}\right)^{-1}\right] \\
-0.6404 \varepsilon-0.1=0
\end{array}
$$

Thus, subject to the constraints (25), (26) and (41), we can choose

$$
q_{11}=1.9106, \quad \varepsilon=0.8286 .
$$

Hence, (53) implies that

$$
q_{22}=0.8700
$$

Using the results provided in the previous section, we can obtain the important matrices as follows:

$$
\begin{array}{ll}
R=\left[\begin{array}{cc}
2.2560 & 0 \\
0 & 1.2905
\end{array}\right], \quad \Omega=\left[\begin{array}{cc}
0.4857 & -0.0992 \\
-0.0992 & 0.1350
\end{array}\right] \\
Y=\left[\begin{array}{cc}
0 & 0 \\
0.1172 & -0.3483
\end{array}\right], \quad Z=\left[\begin{array}{cc}
0 & 0 \\
-0.0948 & 0.3550
\end{array}\right] .
\end{array}
$$

Let us now consider the analytical expression (44). In this case, since

$$
I-B^{+} B=0
$$

the matrix $\Psi$ does not affect the solution. Hence, substituting $T=1$ and $T=-1$ into this expression leads to the following two desired controller gains:

$$
\begin{aligned}
& K_{1}=\left[\begin{array}{ll}
-2.1774 & -0.1239
\end{array}\right] \\
& K_{2}=\left[\begin{array}{ll}
-2.9883 & -0.4846
\end{array}\right] .
\end{aligned}
$$

It is not difficult to verify that the specified mean square boundedness as well as the steady-state variance constraint are achieved.

\section{CONCLUSION}

In this paper, the robust control problem has been considered for parameter uncertain discrete-time stochastic systems where there is a nonzero probability of signal being absent in the measurement. This problem has been investigated by assigning an upper bound to the steady-state covariance, and by parameterizing a set of controller gains that could achieve such an upper bound. It has been shown that, the problem is solvable if several matrix inequalities/equations have positive definite solutions. Specifically, the characterization of the desired controller 
gains has been given, where the design flexibility could be utilized to achieve more expected performance requirements. An numerical example has been provided to illustrate the effectiveness of the proposed design approach.

There are still plenty of topics for future research, for example, the maximization of the missing data probability while the controlled process is still guaranteed to be stable, and handling the signal transmission process that is the output of a Markov chain with known transition probabilities. The results will appear in the near future.

\section{ACKNOWLEDGMENT}

The authors would like to thank the associate editor and three anonymous referees for detailed comments and useful suggestions, which has improved the presentation of this paper. Z. Wang also appreciates several helpful discussions with Dr. F. Yang of Fuzhou University, China, who is working on an EPSRC project at Brunel University, Uxbridge, U.K.

\section{REFERENCES}

[1] R. G. Agniel and E. I. Jury, "Almost sure boundedness of randomly sampled systems," SIAM J. Control Optim., vol. 9, pp. 372-384, 1971.

[2] A. Ben-Israel and T. N. E. Greville, Generalized Inverses: Theory and Applications. New York: Wiley, 1974.

[3] J.-M. Chen and B.-S. Chen, "System parameter estimation with input/output noisy data and missing measurements," IEEE Trans. Signal Process., vol. 48, no. 6, pp. 1548-1558, Jun. 2000.

[4] H. Y. Chung and W. J. Chang, "Covariance control with variance constraints for continuous perturbed stochastic systems," Syst. Control Lett., vol. 19, no. 5, pp. 413-417, 1992.

[5] W. L. DeKoning, "Optimal estimation of linear discrete time systems with stochastic parameters," Automatika, vol. 20, pp. 113-115, 1984.

[6] K. Glover, "All optimal hankel-norm approximations of linear multivariable systems and their $L^{\infty}$-error bounds," Int. J. Control, vol. 39, pp. 1115-1193, 1984.

[7] Q. Ling and M. D. Lemmon, "Optimal dropout compensation in networked control systems," in Proc. IEEE Conf. Decision Control, Dec. 2003, pp. 670-675.

[8] J. Lu and R. E. Skelton, "Robust variance control for systems with finitesignal-to-noise uncertainty," Automatika, vol. 36, no. 4, pp. 511-525, 2000.

[9] J. Nilsson, "Real-time control systems with delays," Ph.D. dissertation, Lund Inst. Technol., Lund, Sweden, 1998.

[10] S. Meerkov and T. Runolfson, "Aiming control," in Proc. IEEE Conf. Decision Control, 1986, pp. 494-498.

[11] N. E. Nahi, "Optimal recursive estimation with uncertain observations," IEEE Trans. Inf. Theory, vol. 15, no. 7, pp. 457-462, Jul. 1969.

[12] W. NaNacara and E. Yaz, "Recursive estimator for linear and nonlinear systems with uncertain observations," Signal Process., vol. 62, pp. 215-228, 1997.

[13] Y. Rosen and B. Porat, "Optimal ARMA parameter estimation based on the sample covariances for data with missing observations," IEEE Trans. Inf. Theory, vol. 35, pp. 342-349, Mar. 1989.

[14] A. Saberi, P. Sannuti, and B. M. Chen, $\mathrm{H}_{2}$ Optimal Control. Englewood Cliffs, NJ: Prentice-Hall, 1995, Series in Systems and Control Engineering.

[15] A. V. Savkin and I. R. Petersen, "Robust filtering with missing data and a deterministic description of noise and uncertainty," Int. J. Syst. Sci., vol. 28, no. 4, pp. 373-390, 1997.

[16] A. V. Savkin, I. R. Petersen, and S. O. R. Moheimani, "Model validation and state estimation for uncertain continuous-time systems with missing discrete-continuous data," Comput. Elect. Eng., vol. 25, no. 1, pp. 29-43, 1999.

[17] J. Schafer, Analysis of Incomplete Multivariate Data. London, U.K.: Chapman \& Hall, 1997.

[18] B. Sinopoli, "Kalman filtering with intermittent observations," in Proc. IEEE Conf. Decision Control, Dec. 2003, pp. 701-708.
[19] R. E. Skelton, T. Iwasaki, and K. M. Grigoriadis, A Unified Algebraic Approach to Linear Control Design. New York: Taylor \& Francis, 1997.

[20] R. F. Stengel, Stochastic Optimal Control: Theory and Application. New York: Wiley, 1986.

[21] Y. Wang, L. Xie, and C. E. de Souza, "Robust control of a class of uncertain nonlinear systems," Syst. Contr. Lett., vol. 19, pp. 139-149, 1992.

[22] Z. Wang, X. Chen, and Z. Guo, "Controller design for continuous systems with variance and circular pole constraints," Int. J. Syst. Sci., vol. 26, no. 5, pp. 1249-1256, 1995.

[23] Z. Wang, D. W. C. Ho, and X. Liu, "Variance-constrained filtering for uncertain stochastic systems with missing measurements," IEEE Trans. Autom. Control, vol. 48, no. 7, pp. 1254-1258, Jul. 2003.

[24] Z. Wang and B. Huang, "Robust $H_{2} / H_{\infty}$ filtering for linear systems with error variance constraints," IEEE Trans. Signal Process., vol. 48, no. 8, pp. 2463-2467, Aug. 2000.

[25] Z. Wang and H. Unbehauen, "Robust $H_{2} / H_{\infty}$ state estimation for systems with error variance constraints: The continuous-time case," IEEE Trans. Autom. Control, vol. 44, no. 5, pp. 1061-1065, May 1999.

[26] — , "Robust $H_{\infty}$ control for systems with time-varying parameter uncertainty and variance constraints," Cybern. Syst., vol. 31, pp. 175-191, 2000.

[27] Z. Wang, J. Zhu, and H. Unbehauen, "Robust filter design with timevarying parameter uncertainty and error variance constraints," Int. J. Control, vol. 72, no. 1, pp. 30-38, 1999.

[28] L. Xie and Y. C. Soh, "Robust Kalman filtering for uncertain systems," Syst. Control Lett., vol. 22, pp. 123-129, 1994.

[29] G. Zhu, K. Grigoriadis, and R. E. Skelton, "Covariance control design for the HST," AIAA J., vol. 18, no. 2, pp. 230-236, 1995.

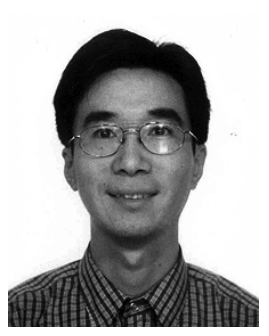

Zidong Wang (SM'02) was born in Jiangsu, China, in 1966. He received the B.Sc. degree in mathematics from Suzhou University, Suzhou, China, in 1986, and the M.Sc. degree in applied mathematics and the Ph.D. degree in electrical engineering, both from Nanjing University of Science and Technology, Nanjing, China, in 1994 and 1990, respectively.

He was appointed as Lecturer in 1990 and Associate Professor in 1994, Nanjing University of Science and Technology. From January 1997 to December 1998, he was an Alexander von Humboldt Research Fellow with the Control Engineering Laboratory, Ruhr-University Bochum, Germany. From January 1999 to February 2001, he was a Lecturer with the Department of Mathematics, University of Kaiserslautern, Germany. From March 2001 to July 2002, he was a University Senior Research Fellow with the School of Mathematical and Information Sciences, Coventry University, U.K. In August 2002, he joined the Department of Information Systems and Computing, Brunel University, Uxbridge, U.K., as a Lecturer, and was promoted to Reader in September 2003. He also holds a TePin Professorship at Donghua University, Shanghai, China. His research interests include dynamical systems, signal processing, bioinformatics, control theory and applications. Since 1997, he has published more than 50 papers in prestigious international journals as the principal author. He is currently serving as an Associate Editor for the International Journal of Systems Science and Circuits, Systems and Signal Processing.

Dr. Wang was awarded the Humboldt research fellowship from the Alexander von Humboldt Foundation in 1996, the JSPS Research Fellowship in 1998 from the Japan Society for the Promotion of Science, and the William Mong Visiting Research Fellowship in 2002 from the University of Hong Kong. He was a recipient of the Outstanding Science and Technology Development Award twice in 1997 from the National Education Committee of China, once in 1997 from the Military Industry General Company of China, once in 1997 from Jiangsu Province Government of China, and once in 1999 from the National Education Ministry of China. He is a Fellow of the Royal Statistical Society. Since 2001, he has served as a member of program committee for more than 20 international conferences, and a very active reviewer for many international journals. He was nominated as an outstanding reviewer for IEEE TRANSACTIONS ON AUTOMATIC CONTROL in 2004 and for the journal Automatica in 2000. He is currently serving as an Associate Editor for IEEE Transactions on Signal Processing, IEEE Transactions on Systems, MAN, AND CYBERNETICS-PART C, IEEE TRANSACTIONS ON CONTROL Systems TeChNOLOGY, and on the Conference Editorial Board for the IEEE Control Systems Society. 


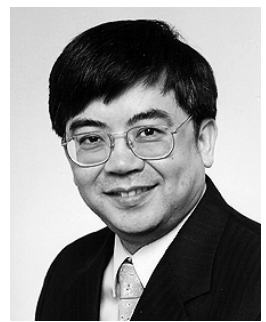

Daniel W. C. Ho (SM'05) received the B.Sc., M.Sc., and Ph.D. degrees in mathematics from the University of Salford, Salford, U.K., in 1980, 1982, and 1986, respectively.

From 1985 to 1988, he was a Research Fellow, Industrial Control Unit, University of Strathclyde, Glasgow, Scotland. In 1989, he joined the Department of Mathematics, City University of Hong Kong, where he is currently an Associate Professor. $\mathrm{He}$ is an Associate Editor of Asian Journal of Control. His research interests include H-infinity control theory, robust pole assignment problems, adaptive neural wavelet identification, and nonlinear control theory.

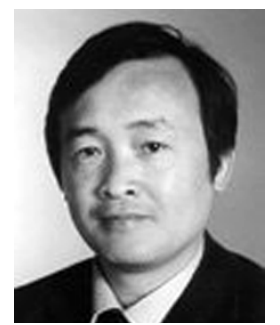

Xiaohui Liu received the B.Eng. degree in computing from Hohai University, Nanjing, China, in 1982 and the Ph.D. degree in computer science from Heriot-Watt University, Edinburgh, U.K., in 1988.

Between October 1987 and October 1989, he was a Member of Research Staff of the Department of Electrical Engineering with both Heriot-Watt University and Durham University, Durham, U.K. He then joined the Department of Computer Science, Birkbeck College, University of London, London, U.K., for 11 years, first as a Lecturer and, subsequently, a Senior Lecturer. Since October 2000, he has been with the Department of Information Systems and Computing, Brunel University, Uxbridge, U.K., where he is presently Professor of Computing and Head of the Intelligent Data Analysis Group, conducting interdisciplinary research involving artificial intelligence, dynamic systems, signal processing, and statistics. He has published more than 80 journal articles/book chapters/conference publications in these areas. He serves on the editorial/review boards of the Applied Intelligence Journal, International Journal of Information Technology and Decision Making, and the Intelligent Data Analysis Journal.

Dr. Liu founded the biennial Intelligent Data Analysis conference series in 1995 and has been on the technical program committees of various international conferences. He has been a principal investigator of numerous research projects funded by research councils, charities and industries. In 2002, he gave a keynote address at the International Conference of the Royal Statistical Society in the U.K. 